\title{
Infrared Photocoagulation Therapy
}

National Cancer Institute

\section{Source}

National Cancer Institute. Infrared Photocoagulation Therapy. NCI Thesaurus. Code C62730.

The use of an intense beam of light, such as a laser, to seal off blood vessels or destroy tissue. 